\title{
Gravitational Waves: A 100-Year Tool Applied to the Dark Energy Problem
}

\author{
M. Soares-Santos ${ }^{1}$, S. V. B. Gonçalves ${ }^{2}$, J. C. Fabris ${ }^{2}$, and E. M. de Gouveia Dal Pino ${ }^{1}$ \\ ${ }^{1}$ Instituto de Astronomia, Geofísica e Ciências Atmosféricas, Universidade de São Paulo, \\ R. do Matão 1226, Cidade Universitária, São Paulo, SP, 05508-090, Brazil \\ 2 Departamento de Física, Universidade Federal do Espírito Santo, \\ Av. Fernando Ferrari s/n, Vitória, ES 29075-910, Brazil
}

(Received on 14 October, 2005)

\begin{abstract}
Recent observations from type Ia Supernovae and from cosmic microwave background (CMB) anisotropies have revealed that most of the matter of the Universe interacts in a repulsive manner, composing the so-called dark energy constituent of the Universe. Determining the properties of dark energy is one of the most important tasks of modern cosmology and this is the main motivation for this work. The analysis of cosmic gravitational waves $(\mathrm{GW})$ represents, besides the $\mathrm{CMB}$ temperature and polarization anisotropies, an additional approach in the determination of parameters that may constrain the dark energy models and their consistence. In recent work, a generalized Chaplygin gas model was considered in a flat universe and the corresponding spectrum of gravitational waves was obtained. In the present work we have added a massless gas component to that model and the new spectrum has been compared to the previous one. The Chaplygin gas is also used to simulate a $\Lambda$-CDM model by means of a particular combination of parameters so that the Chaplygin gas and the $\Lambda$-CDM models can be easily distinguished in the theoretical scenarios here established. We find that the models are strongly degenerated in the range of frequencies studied. This degeneracy is in part expected since the models must converge to each other when some particular combinations of parameters are considered.
\end{abstract}

\section{INTRODUCTION}

The wave-like solutions of the Einstein equations - the socalled gravitational waves - are among the most outstanding predictions of the general relativity and one can actually say the gravitational radiation represents a new window, besides its electromagnetic analog, to the astrophysical and cosmological observations. Due to the lack of direct detection one cannot say this window is already open, but great efforts have been done in order to overcome such difficulty and a large amount of theoretical work (the present one included) has been motivated by these good perspectives.

As soon as this new window is open, one could use the gravitational wave signal to obtain extra information about the very same problems up to now investigated only in the electromagnetic band and the aim of this work is to stablish weather this potential tool could be applied to the trickiest problem of modern cosmology: the dark energy.

A large number of dark energy candidate models have been proposed since that the SNeIa [1-3] and CMB [4] experiments revealed the accelerating expansion of the universe. In order to explain the observational data all these models consider an equation of state with negative pressure. The cosmological constant models consider a simple equation of state, $p=-\rho$, which however results a huge discrepancy with the data [5]. Another class of models considers a scalar field, called quintessence [6], a third class assumes a perfect fluid with a negative pressure which is proportional to the inverse of the energy density, the Chaplygin gas [7], and finally a forth class, considers an $X$-fluid with an equation of state $p=\omega \rho$, where $\omega$ is a negative constant, known as phantom energy when $|\omega|>1$ [5].

Until the present the combined observations have been unable to determine the most appropriate description, but gravitational waves represent a potential tool that may - in near future - offer additional constraints to the cosmological para- meters and distinguish among the models.

In this paper, we study the spectrum of gravitational waves due to the X-fluid model and compare the results with a previous work [8], where the cosmological constant and the generalized Chaplygin gas models were taken into account. In section §II these models are described; in §III the GW equations are presented. The spectra analysis is left to $\S I V$, which is followed by the conclusions of this work.

\section{OUTLINE OF THE MODEL}

We consider a flat, homogeneous and isotropic universe described by the Friedman-Robertson-Walker metric, which makes the Einstein's equations to assume the form

$$
\begin{gathered}
\left(\frac{\dot{a}}{a}\right)^{2}=\frac{8 \pi G}{3}\left(\rho_{m}+\rho_{d e}\right), \\
2 \frac{\ddot{a}}{a}+\left(\frac{\dot{a}}{a}\right)^{2}=-8 \pi G\left(p_{m}+p_{d e}\right),
\end{gathered}
$$

where $a$ is the scale factor of the universe, while $\rho_{m}$ and $\rho_{d e}$ are the pressureless fluid and the dark energy densities, respectively. The pressures $p_{m}$ and $p_{d e}$ of the fluids are related with their densities by the equations of state $p_{m}=0$, and $p_{x}=\omega \rho_{x}$, with $\omega<0$ (in the case of an X-fluid) or $p_{c}=-A / \rho_{c}^{\alpha}$, with $A, \alpha>0$ (in the case of the Chaplygin gas), respectively. We take the scale factor today as the unity, $a_{0}=1$ (the subscripts 0 , according to the current notation, indicate the present values of the quantities) and rewrite the dark energy density for both cases as:

$$
\begin{gathered}
\rho_{x}=\rho_{x_{0}} a^{-3(\omega+1)}, \\
\rho_{c}=\rho_{c_{0}}\left[\bar{A}+\frac{(1-\bar{A})}{a^{3(\alpha+1)}}\right]^{\frac{1}{\alpha+1}}, \bar{A}=\frac{A}{\rho_{c_{0}}^{\alpha+1}} .
\end{gathered}
$$


With equations (3) and (4), we write the Einstein's equations in the form

$$
\begin{gathered}
\frac{\dot{a}}{a}=H_{0}\left(\frac{\Omega_{m_{0}}}{a^{3}}+\frac{\Omega_{x_{0}}}{a^{3(\omega+1)}}\right)^{1 / 2} \\
\frac{\ddot{a}}{a}=-\frac{1}{2} H_{0}^{2}\left(\frac{\Omega_{m_{0}}}{a^{3}}+\Omega_{x_{0}} \frac{1+3 \omega}{a^{3(\omega+1)}}\right),
\end{gathered}
$$

for the X-fluid case, and

$$
\begin{array}{r}
\frac{\dot{a}}{a}=H_{0}\left[\frac{\Omega_{m_{0}}}{a^{3}}+\Omega_{c_{0}}\left(\bar{A}+\frac{1-\bar{A}}{a^{3(\alpha+1)}}\right)^{\frac{1}{\alpha+1}}\right]^{1 / 2}, \\
\frac{\ddot{a}}{a}=H_{0}^{2}\left[-\frac{\Omega_{m_{0}}}{2 a^{3}}+\Omega_{c_{0}}\left(\bar{A}+\frac{1-\bar{A}}{a^{3(\alpha+1)}}\right)^{\frac{1}{\alpha+1}} \times\right. \\
\left.\left(1-\frac{3(1-\bar{A})}{2 a^{3(\alpha+1)}}\left(\bar{A}+\frac{1-\bar{A}}{a^{3(\alpha+1)}}\right)^{-1}\right)\right],
\end{array}
$$

for the Chaplygin gas. The Hubble constant $H_{0}$ is defined as $H_{0}=\dot{a}_{0} / a$ and, once we are restricted to a flat universe, the fractions of pressureless matter and dark energy gas today, $\Omega_{m_{0}}$ and $\Omega_{d e_{0}}$, respectively, obey the relation $\Omega_{m_{0}}+\Omega_{d e_{0}}=1$.

With these two last equations we are able to write the GW amplitude differential equation as a function of the observable parameters $H_{0}, a, \Omega_{m_{0}}$ and $\Omega_{d e_{0}}$, and of the dark energy fluid parameters: $\bar{A}, \alpha$ or $\omega$. It is also important to remark that

(i) if $\bar{A}=0$, then the Chaplygin gas behaves like the pressureless fluid and the situation is the same as if we had set $\Omega_{m_{0}}=1$;

(ii) on the other hand, for $\bar{A}=1$ it behaves like the cosmological constant and therefore, we recover the $\Lambda$-CDM scenario; (iii) the $\mathrm{X}$-fluid is equivalent to the cosmological constant for $\omega=-1$.

Taking into account the above remarks we use different sets of parameters to compare three classes of models - cosmological constant, X-fluid and generalized Chapligyn gas - and test how they affect the evolution of the cosmic GW background.

\section{GW EQUATIONS}

Cosmological gravitational waves are obtained through little perturbations $h_{i j}$ on the metric. Hence, the tensor $g_{i j}^{(0)}$, related to the unperturbed metric, is replaced by $g_{i j}=g_{i j}^{(0)}+h_{i j}$ and the resulting expression is [9] :

$$
\ddot{h}-\frac{\dot{a}}{a} \dot{h}+\left(\frac{k^{2}}{a^{2}}-2 \frac{\ddot{a}}{a}\right) h=0,
$$

where $k$ is the comoving wave number times the velocity of light $(k=2 \pi c / \lambda)$, the dots indicate time derivatives and $h(t)$ is defined as: $h_{i j}(\vec{x}, t)=h(t) Q_{i j}$, where $Q_{i j}$ are the eigenmodes of the Lagrangian operator, such that $Q_{i i}=\partial_{k} Q_{k i}=0$.
Proceeding with a variable transformation from time to the scale factor $a$, and representing the derivatives with respect to $a$ by primes, equation (9) assumes the form

$$
h^{\prime \prime}+\left(\frac{\ddot{a}}{\dot{a}^{2}}-\frac{1}{a}\right) h^{\prime}+\frac{1}{\dot{a}^{2}}\left(\frac{k^{2}}{a^{2}}-2 \frac{\ddot{a}}{a}\right) h=0
$$

and, from the substitution of the background equations (7) and (8), or (5) and (6), which concern to the dark energy fluid, into (10), one can easily express $h$ in terms of the parameters of the model.

\section{A. Generalized Chaplygin gas}

Let us perform the last operation mentioned above and, in order to find $h$ as a function of the redshift $z$, let us (i) use the known relations $1+z=\frac{a_{0}}{a}, a_{0}=1$; (ii) perform a second change of variables (from $a$ to $z$ ); and (iii) take back the dots to indicate, from now on, the new integration variable. These operations result in

$$
\begin{gathered}
{\left[\frac{2}{1+z}+\frac{3}{2}(1+z)^{2} \frac{f_{1}}{f_{2}}\right] \dot{h}+} \\
{\left[\frac{k^{2}}{f_{2}}-\frac{2}{(1+z)^{2}}+3(1+z) \frac{f_{1}}{f_{2}}\right] h=0,} \\
f_{1}=\Omega_{m_{0}}+\Omega_{c_{0}}(1-\bar{A})(1+z)^{3 \alpha} \times \\
{\left[\bar{A}+(1-\bar{A})(1+z)^{3(\alpha+1)}\right]^{\frac{1}{\alpha+1}-1},} \\
f_{2}=\Omega_{m_{0}}(1+z)^{3}+\Omega_{c_{0}} \times \\
{\left[\bar{A}+(1-\bar{A})(1+z)^{3(\alpha+1)}\right]^{\frac{1}{\alpha+1}},}
\end{gathered}
$$

where $\mathrm{k}$ is redefined to absorb the Hubble constant, i.e., $k=$ $2 \pi c / H_{0} \lambda$ and, therefore, $h$ is a dimensionless quantitiy.

\section{B. X-fluid}

To obtain the equivalent equations for the X-fluid model, we use the same procedure described above. The result may be written in the same form as in (11), with $f_{1}$ and $f_{2}$ redefined as:

$$
\begin{gathered}
f_{1}=\Omega_{m_{0}}+\Omega_{x_{0}}(1+\omega)(1+z)^{3 \omega}, \\
f_{2}=\Omega_{m_{0}}(1+z)^{3}+\Omega_{x_{0}}(1+z)^{3(\omega+1)} .
\end{gathered}
$$

One can easily notice that, if $\omega=-1$ and $\bar{A}=1$, both cases converge to the same equation and then reproduce the $\Lambda$-CDM scenario. 


\section{GW SPECTRA ANALYSIS}

The power spectrum of gravitational waves, defined as

$$
P \equiv\left(\frac{d \Omega_{G W}}{d \ln v}\right)^{1 / 2}=\left|h_{0}(v)\right| v^{5 / 2}
$$

[where $h_{0}(v)=h(0)$ and $v=H_{0} k / 2 \pi$ ], is generally obtained from the solutions of (11) which are found by means of a numerical algorithm specifically created for this problem. In the particular case of the $\Lambda$-CDM model, an analytical result is also possible and this fact is used to verify the accuracy of the calculation.

For each of the cases of interest, we have assigned some common parameters, namely the initial conditions $h\left(z_{i}\right)=$ $v^{-3} 10^{-5}, \dot{h}\left(z_{i}\right)=v^{-2} 10^{-5}, z_{i}=4000$; the range of frequencies $10^{-18} \mathrm{~Hz} \leq \mathrm{v} \leq 10^{-15} \mathrm{~Hz}$, which is a small part of the range of observational interest for $\mathrm{GW}$ that goes up to $10^{10} \mathrm{~Hz}$; and the normalization constant imposed by the CMB [10]

$$
\left.\frac{d \Omega_{G W}}{d \ln v}\right|_{10^{-18}} \leq 10^{-10} .
$$

The resulting spectra have all a similar behavior: they are strongly oscillating, but with an increasing amplitude, as indicated in the figures below.

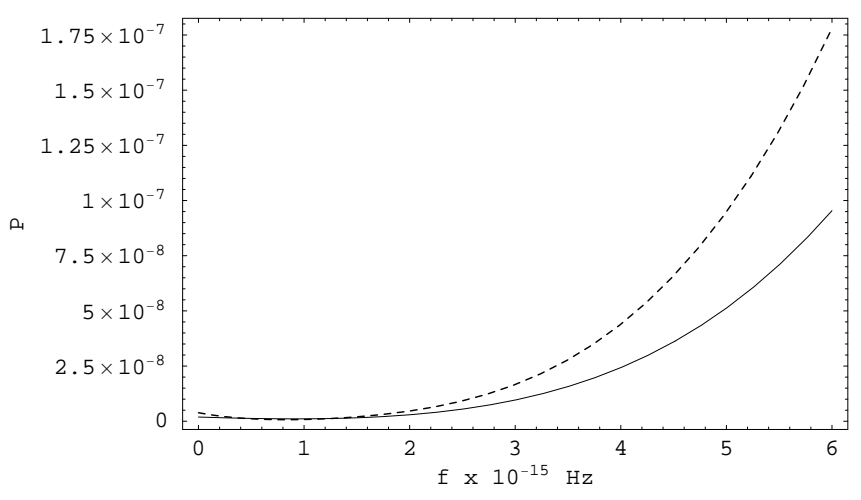

Figure 1. Comparison of GW spectra due to the Einstein-de Sitter (solid line) model and the X-fluid model, with $\Omega_{x_{0}}=0.7$ and $\Omega_{m_{0}}=0.3$ (dashed line). The latter is not sensitive to the parameter $\omega$.

¿From Figure 1 one can notice that the X-fluid class of models is completely degenerated (dashed line), i.e., the models with cosmological constant $(\omega=-1)$, phantom energy $(\omega<-1)$, and the intermediate cases $(-1<\omega<0)$ are indistinguishable. It is worthwhile to remark however that the dark energy component (70\% of the total energy density) leads to a faster-increasing spectrum compared to the Einstein-de Sitter $\left(\Omega_{m_{0}}=1\right)$ case.

The spectra produced by the Chaplygin gas model is represented in Figures 2 and 3, where two important features are straightforward. The first is that this model plays the role of a unified dark energy-matter fluid, since the curves with $\Omega_{c_{0}}=0.7$ and $\Omega_{c_{0}}=0.97$ (cf. Figure 2) are coincident.

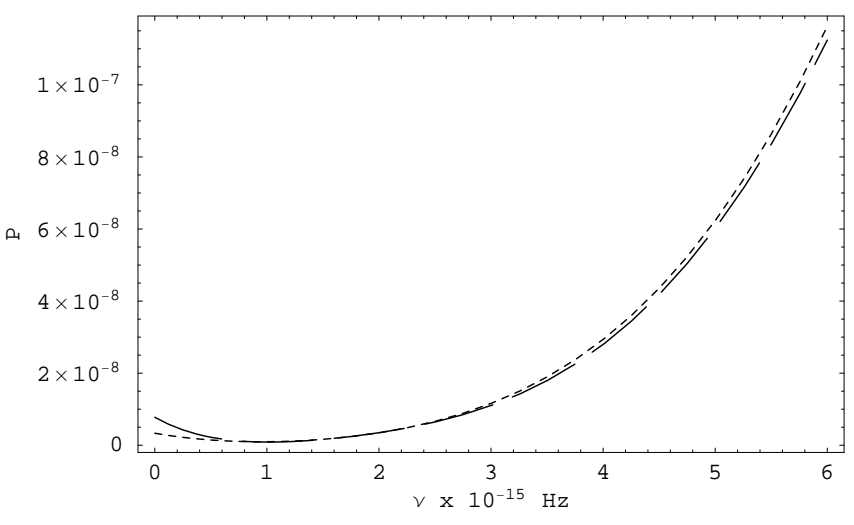

Figure 2. GW spectrum for the Chapligyn gas model with $\Omega_{c_{0}}=0.7$ (long dashed line) and $\Omega_{c_{0}}=0.97$ (short dashed line) showing its behavior as a unified dark energy-matter model.

The second is that the Chaplygin gas interpolates the dust and cosmological constant fluids: the region allowed for the curves due to this model is bounded by the cosmological constant and the matter-only curves.

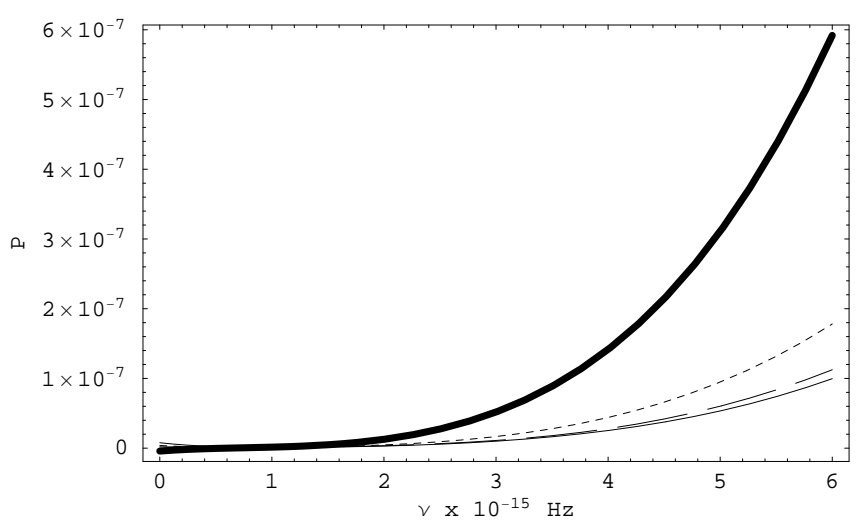

Figure 3. Four models compared: de Sitter (thick solid line), Einstein-de Sitter (thin solid line), Chapligyn gas with $\alpha=1$ and $\bar{A}=0.5$ (long-dashed line) and $\Lambda$-CDM or Chapligyn gas with $\bar{A}=1$ (short dashed line).

Not so straightforward to interpret is the fact that a fasterincreasing spectrum is obtained here not with larger values of dark-energy densities, but with larger values of the parameter $\bar{A}$. This means that the Chaplygin gas behaves like a mixture of cosmological constant and matter: as $\bar{A}$ tends to unity, the cosmological constant dominates de mixture and therefore the spectrum tends to its upper bound; on the other hand, as $\bar{A}$ tends to zero, the matter component dominates and the spectrum becomes similar to the Einstein-de Sitter one, which is a fixed lower bound. The upper bound is not fixed though. It can change with the variation of the density parameter and the last upper boundary is fixed by the de Sitter curve (thick solid line in Figure 3). The long-dashed line in Figure 3, for instance, represents the case where $\bar{A}=0.5$ and it lays between the $\Lambda$-CDM (with $\Omega_{\underline{c}_{0}}=0.7$ ) and the Einstein-de Sitter lines and, as we increase $\bar{A}$ it tends to the short-dashed line, which is the upper limit for $\Omega_{c_{0}}=0.7$.

Actually, since we (i) cannot distinguish between $\Lambda$ and the other X-CDM cases (as discussed above), and (ii) can easilly reproduce the corresponding curve with an appropriate com- 
bination of the parameters $\bar{A}$ and $\Omega_{c_{0}}$, the Chaplygin gas and the X-fluid models are partially degenerated.

A criterious reader must have noticed we said any word about the parameter $\alpha$. The reason for this silence is that no dependence on $\alpha$ was found in our previous works $[8,11]$ on this subject. The spectrum presents no significative differences for $0 \leq \alpha \leq 1$, in the frequency range studied.

\section{CONCLUSIONS}

In this work we have compared three important dark energy models in the context of the gravitational wave spectra that they should be able to induce and we observe that the models are strongly degenerated in the range of frequencies studied - from $10^{-18}$ up to $10^{-15} \mathrm{~Hz}$. In addition, for the Chaplygin gas, the negligible dependence on the density parameter $\Omega_{c_{0}}$ is consistent with the idea of a unified dark component $[7,8]$. Since the gas interpolates both dark energy and matter, the suppression of the dark matter density parameter (see, Figure 2) should not affect the spectrum, as observed. The X-fluid component contribution seems to be completely degenerated and must be better studied in the near future, specially at larger frequency ranges.

Unfortunately, no direct observational data from cosmological gravitational waves is available up to now, but still it is important to investigate the relation between these gravitational waves produced or amplified by the presence of the dark energy component and the CMB temperature and polarization anisotropies which are already observationally determined [12]. This correlation between CMB and gravitational waves will be properly studied once we have a larger range of frequencies in the GW spectra.

\section{Acknowledgments}

M.S.S. and E.M.G.D.P. acknowledge financial support from the Brazilian Agencies FAPESP and CNPq.
[1] A. G. Riess et al., Astron. Journal 116, 1009 (1998).

[2] S. Permultter et al., Nature 391, 51 (1998).

[3] J. L. Tonry et al., Astroph. Journal 594, 1 (2003).

[4] M. Tegmark et al., Phys. Rev. D 69, 103501 (2004).

[5] V. Sahni, astro-ph/0403324.

[6] R. R. Caldwell, and M. Doran, Phys. Rev. D 69, 103517 (2004).

[7] V. Gorini, A. Kamenshchik, U. Moschella, and V. Pasquier, $\mathrm{gr}=\mathrm{qc} / 0403062$.

[8] J. C. Fabris, S. V. B. Gonçalves, and M. S. Santos, Gen. Rel. Grav. 36, 2559(2004).
[9] S. Weinberg, Gravitation and Cosmology, John Wiley, New York, 1972

[10] A. Riazuelo and J. P. Uzan, Phys. Rev. D 62, 083506 (2000)

[11] M. Soares-Santos, S. V. B. Gonçalves, J. C. Fabris, and E. M. de Gouveia Dal Pino, Modeling the spectrum of gravitational waves in the primordial Universe, in AIP Conf. Proc. 784, 800 (2005).

[12] Bennett et al, Astroph. Journal Suppl. 148, 1 (2003). 